\title{
The Significance of Streamlined Postprocessing Approaches for Clinical fMRI
}

T he article in this issue of the American Journal of Neuroradiology (AJNR) entitled "Simple fMRI Postprocessing Suffices for Normal Clinical Practice" 1 is an important contribution to the current blood oxygen level-dependent (BOLD) fMRI literature because it highlights an emerging trend in functional neuroimaging software development that will likely promote increasing clinical use of fMRI in the near future. Although many attempts have been made during the past decade to standardize fMRI paradigms across institutions, streamline BOLD fMRI preprocessing and postprocessing, validate fMRI by using intraoperative electrophysiologic criterion standards, and establish standards for fMRI image acquisition, processing, and interpretation, only some of these have been reasonably successful. ${ }^{2-4}$ The greatest strength of the BOLD fMRI technique-its immense versatility with respect to assessment of cognitive networks, paradigm design, and signalprocessing approaches- has also proved to be its greatest weakness from a clinical standardization standpoint.

Presurgical planning for resectable brain lesions was the original clinical application of BOLD fMRI and remains the sole widely accepted clinical application (if we consider the broader category of pretherapeutic planning, including planning for radiation therapy and hemispheric language lateralization for epilepsy) for which Current Procedural Terminology codes were established in 2007. ${ }^{5}$ Currently, expansion of clinical indications to include posttherapeutic monitoring of eloquent cortex has also been accepted. In a nutshell, clinical functional MR imaging enables neuroradiologists to go beyond mere characterization of anatomic findings and instead provide critical functional-anatomic correlation that is essential for accurate assessment of the risks of neurosurgical or radiation treatment.

The relatively cumbersome nature of research-level BOLD fMRI postprocessing software (which typically requires graduatelevel experience in image processing, including computer programming within environments such as Matlab [MathWorks, Natick, Massachusetts] for generation of custom-made scripts for semiautomated execution of multiple processing steps for clinical applications) has been a limitation recently overcome by more streamlined commercially available FDA-approved packages. These newer packages allow more user-friendly interaction; better image overlays, including interactive $3 \mathrm{D}$ viewing; and greater compatibility with PACS servers and neuronavigation software for facilitation of image export and viewing by referring physicians and patients alike. ${ }^{6}$

These developments have led to the capability of importing functional images into the operating room in a fashion that neurosurgeons can easily use in planning their surgery. With brain shift occurring following violation of the dura, the original exact landmarks provided by the preoperative functional imaging may not be accurate. Overlays with postgadolinium anatomic images allow neurosurgeons to rely on venous and gyral anatomic landmarks that are useful even as resection progresses. This is particularly true when higher field (1.5T and 3T) intraoperative MR imaging systems that allow nearly real-time monitoring of the extent of lesion resection are used.

Of course, these advances have certainly not made the use of research software obsolete because the relatively "turnkey" semiautomated processing available with the commercial software packages has many limitations that, in many cases, need to be addressed by additional processing by using more sophisticated research packages. The reason is that such powerful research software allows alteration of many preset default parameters (that cannot be adjusted with the more streamlined commercial packages) that may not always be optimal for every clinical case. Sometimes clinical interpretation may be dependent on additional information that the streamlined packages may not be able to provide. An example provided in this article is the need for quantitative assessment of suprathreshold-activated voxels in clusters of interest for laterality index computation. In other words, while the commercial packages may allow qualitative assessment of hemispheric lateralization, they frequently do not allow voxeland cluster-level quantitative assessments that would be necessary for accurate computation of laterality indices or even determination of centroids (or centers of mass) of activation on the basis of both individual voxel $t$ values and spatial extent of suprathreshold voxels within a cluster or group of adjacent clusters. Another example would be the need to vary spatial-extent thresholds (ie, clustering thresholds) at a given $P$ value or $t$ statistic threshold after analysis by using a general linear model (GLM) approach. 
Another example would be an instance in which a more complex paradigm design is used with multiple contrasts or varying epoch lengths, in which a standard dual-contrast-equivalent epoch block-design analysis approach (the typical default approach used by most commercial software) would not be adequate.

Most real-time fMRI algorithms and many commercial offline postprocessing packages generally make the assumption that dual contrast block-design paradigms are used with equivalent duration of the activation and control epochs; thus, these will fail to provide reliable activation maps in cases of more complex paradigm design. Still another example would be the need to use approaches other than a simple $t$ test, GLM, or cross-correlation method in cases in which parallel cognitive processes may display different time courses (eg, an independent-component-analysis approach may be more appropriate in such circumstances, but the commercially available software typically cannot handle such analyses).

Nevertheless, despite these limitations, the streamlined software has changed the landscape for clinical functional imaging in many ways. One such change includes the widespread availability of real-time fMRI, which has enhanced the overall success rates of clinical fMRI examinations by enabling neuroradiologists to make real-time informed decisions to repeat poor runs of a task that may have otherwise resulted in a nondiagnostic examination. Some studies have also shown that the real-time maps may be comparable, for both functional localization and lateralization, with those obtained through traditional more elaborate postprocessing approaches. ${ }^{7,8}$

Nevertheless, with greatly improved quality control (QC) analytic tools built into such commercially available software, including rotational and translational head-motion plots and, in some cases, even more sophisticated physiologic monitoring techniques used during BOLD EPI data acquisition, these newer packages are beginning to rival the old research packages in scope and clinical utility. Some of these software packages are provided by MR imaging scanner vendors, as in this particular article in the current issue, while others are provided by different vendors. In particular, the advent of fully integrated FDA-approved clinical fMRI systems during the past 5 years represents an extremely important development that has resulted in greater ease of clinical fMRI practice and has opened the door to clinical fMRI for a larger number of academic medical centers and even for a growing number of radiology private practice groups in the United States. ${ }^{5}$

Such integrated systems have replaced older less reliable research systems that had to be assembled part by part through the purchase of individual components from separate vendors. Such components include stimulus-presentation software and hardware; scanner-triggering software; multimedia projectors or video goggles; mirrors; response keypads; in some cases, fiber optic gloves or other sensor devices; eye-tracking equipment; and respiratory belts, depending on individual center research applications. The newer integrated systems include all necessary basic components and provide relatively seamless integration of components and available customer support as well as integration with PACS and neuronavigation systems for reliable and streamlined postprocessed image export.
Ongoing effort at the national level, including work currently being conducted by the Quantitative Imaging Biomarkers Alliance Committee of the Radiological Society of North America ${ }^{9}$ is addressing the need for standardization in the image acquisition, pre- and postprocessing, and interpretation steps in the clinical fMRI workflow. Furthermore, the American Society of Functional Neuroradiology (ASFNR) is in the process of launching a multicenter trial at this writing to explore the impact of presurgical functional imaging on surgical planning and to simultaneously attempt to standardize motor and language paradigms across institutions. In addition, a taskforce of the American College of Radiology, in collaboration with the American Society of Neuroradiology and the Society for Pediatric Radiology, has established a new Practice Guideline for Performance of Functional Magnetic Resonance Imaging of the Brain in 2012. ${ }^{10}$ Last, in 2011 the ASFNR established formal BOLD fMRI Dictation Guidelines to help further standardize the practice of clinical fMRI by providing clear recommendations for reporting of results of these examinations. $^{11}$

MR imaging scanner vendors and smaller companies that offer integrated clinical fMRI systems as described above have adapted their products to meet these new standards and are thus paving the road for greater clinical use of BOLD fMRI at centers across the nation.

One potential criticism of the article included in this issue of the AJNR may be that only 1 commercial fMRI processing software package was evaluated and compared with a widely used robust research software package (SPM5; Wellcome Department of Imaging Neuroscience, London, UK). This may potentially introduce some commercial bias into the study and, more important, may not allow generalization of the study results to all currently available streamlined commercial packages. Indeed, among the currently available FDA-approved commercial packages, many differences exist with respect to available analysis approaches, threshold settings, and display features. However, in my opinion, none can match the versatility and depth of the processing capability of powerful research software such as SPM (currently in Version 8), Analysis of Functional NeuroImages (http:// afni.nimh.nih.gov/afni/), or the FMRI of the Brain Software Library (http://www.fmrib.ox.ac.uk/fsl). However, the newer integrated systems offer greater versatility in general than the software provided by MR imaging scanner vendors, in my opinion. Both types of packages offer better compatibility with PACS and neuronavigation software than stand-alone research packages, however; and in some cases, the 3D image fusion and overlay as well as overall visualization capabilities are superior from an enduser (neuroradiologist or neurosurgeon) standpoint. Thus, from a clinical workflow perspective, it is advantageous to use the commercial software.

In actual practice at my center, we generally rely on the more sophisticated research software only as a backup in cases in which we think that the commercial software is not adequate for one reason or another, and the percentage of cases in which such problems are encountered is generally on the order of $5 \%-10 \%$. We also rely on off-line research software for a more detailed QC analysis than our commercial package can offer; for example, we look at imaging volumes containing an exces- 
sive number of data outliers and use these to select the most optimal runs for inclusion in the final analysis when the patient performs $>1$ run of the same task. This is usually the case when we suspect suboptimal activation or excessive head motion on the basis of real-time fMRI maps obtained during patient task performance. We also use the research software in cases in which there is a clinical need to compute a quantitative laterality index rather than simply rely on a qualitative assessment of hemispheric language lateralization. In actual practice, however, this scenario is encountered more often in retrospective research than in actual clinical practice. Thus, overall, we have found the commercial software packages to be mostly satisfactory for clinical use, similar to the findings in this article. Furthermore, the commercial packages have the additional value of having official FDA approval, which may be considered meaningful from a medicolegal standpoint, even though this is of little or no concern from a medical or scientific standpoint.

However, in the broader perspective, the real value of such streamlined software is the impact it can make on clinical workflow, which is perhaps the most important element in the success of a clinical fMRI service in the current era. With such workflow optimization, a complex multiparadigm clinical fMRI examination can be performed, analyzed, interpreted, reviewed, and made available for PACS and neuronavigation in as little as 6 hours, whereas in the past, the same workflow used to take more than several days to accomplish. When one considers the potential difference in neurosurgical management, the small sacrifice paid for minimal loss of research-level versatility is probably well worth making in the interest of improving overall patient care.

\section{REFERENCES}

1. González-Ortiz S, Oleaga L, Pujol T, et al. Simple fMRI postprocessing suffices for normal clinical practice. AJRN Am J Neuroradiol 2013;34:1188-93

2. Bizzi A, Blasi V, Falini A, et al. Presurgical functional MR imaging of language and motor functions: validation with intraoperative electrocortical mapping. Radiology 2008;248:579-89

3. Binder JR, Swanson SJ, Hammeke TA, et al. Determination of language dominance using functional MRI: a comparison with the Wada test. Neurology 1996;46:978-84

4. Stippich C, Rapps N, Dreyhaupt J, et al. Localizing and lateralizing language in patients with brain tumors: feasibility of routine preoperative functional MR imaging in 81 consecutive patients. Radiology 2007;243:828-36

5. Rigolo L, Stern E, Deaver P, et al. Development of a clinical functional magnetic resonance imaging service. Neurosurg Clin N Am 2011;22:307-14, $\mathrm{x}$

6. Pillai JJ. The evolution of clinical functional imaging during the past 2 decades and its current impact on neurosurgical planning. AJNR Am J Neuroradiol 2010;31:219-25

7. Fernández G, de Greiff A, von Oertzen J, et al. Language mapping in less than 15 minutes: real-time functional MRI during routine clinical investigation. Neuroimage 2001;14:585-94

8. Kesavadas C, Thomas B, Sujesh S, et al. Real-time functional MR imaging (fMRI) for presurgical evaluation of paediatric epilepsy. Pediatr Radiol 2007;37:964-74

9. http://qibawiki.rsna.org/index.php?title=FMRI_tech_ctte. Accessed on December 7, 2012

10. http://www.acr.org/Quality-Safety/Standards-Guidelines/PracticeGuidelines-by-Modality/MRI. Accessed on December 14, 2012

11. http://www.asfnr.org/docs/BOLD-fMRI-Dictation-Guidelines.pdf. Accessed on December 7, 2012

\section{J.J. Pillai} Division of Neuroradiology Russell H. Morgan Department of Radiology and Radiological Science The Johns Hopkins Hospital Baltimore, Maryland

http://dx.doi.org/10.3174/ajnr.A3446 